\title{
Controversion on antioxidant administration in elderly
}

\begin{abstract}
In one of the aging process theory states that free radicals are responsible to aging process that occur as a result of damage at the cellular level and the resulting tissue. Normally, there is a dynamics and continuous balance between oxidant/free radicals and antioxidant in the body. If there is an increase in free radicals beyond the body's ability to produce endogenous antioxidants against free radicals activity contribute to the decline of cell function. The speed of damage and declined cell function become the basis of the rapid aging process and the emergence of various degenerative disease. Although there is a lot of antioxidant suplements to inhibit this process but currently the results of various studies are still not satisfying and often inconsistent. Several things which must be considered in studying the effectiveness of antioxidant suplementations. Development of the oxidation mechanism concept that became the basic of aging process. Determine the right marker of oxidative damage and antioxidant. Finding the levels of therapeutic window so the effective dose of antioxidant supplementation can be determined. Understand the knowledge of antioxidant molecules that can turn into pro-oxidant. According to this, the further studies to determine the effectivity of new antioxidant suplementation that classified as an enzymatic antioxidant such as SOD (sodium dismutase). These antioxidants are expected to provide a consistent way to inhibit the aging process and prevent or slow the emergence of degenerative disease.
\end{abstract}

Keywords: antioxidant, genetic, mitochondria, oxidize, hydroperoxyl
Volume 3 Issue 2 - 2018

\section{IGP Suka Aryana,' Ra Tuty Kuswardhani' \\ 'Geriatric Division of Internal Medicine Departement, Medical Faculty of Udayana University/ Sanglah Teaching Hospital, Bali,} Indonesia.

\section{Correspondence: IGP Suka Aryana, Geriatric Division of Internal Medicine Departement, Medical Faculty of Udayana University/ Sanglah Teaching Hospital, Bali, Indonesia, Email aryanasuka@yahoo.com}

Received: April 16, 2018 | Published: May 15, 2018

\section{Introduction}

There are many theories that try to explain the aging process but have not been satisfying. The theory of free radicals is the only theory closest to the basis of the mechanism of the aging process. This theory states the influences of free radicals will be modified by genetic and environtmental factors. Free radicals are responsible for the cell and tissue destruction due to the oxidation process of biological molecules of fat, protein and nucleic acid. In 1972, it was known that mitochondria were responsible for free radicals reaction in cells. Age is determined by the speed of mitochondria destruction by the free radicals. Mitochondria will continue to produce free radicals in its entire life until its mitochondrial DNA is attacked and destroyed resulting in cell death. Cells always use oxygen but carry the consequences of generating the reactive oxygen species (ROS) free radicals and trigger the formation of endogenous antioxidant to neutralize the oxidant effect. ${ }^{1,2}$ In aging process, there are seems to be an imbalance of free radical production and antioxidants defences in a large scale. To inhibit this process requires efforts to decreasing the free radicals production and increasing the antioxidants in the body. This is the basic of studies that try to evaluate the effectiveness of antioxidant suplementation to inhibit the aging process and to prevent the emergence of degenerative disease so that elongating the life expectancy. Studies in western countries still get an inconsistent results. ${ }^{3}$

\section{Oxidative Damage}

Under normal conditions there is a $1 \%$ of ROS daily that is out of control of endogenous antioxidant defenses and will accumulated and plays a role in the aging process. This condition can cause more than one hundred pathological disorder in human body. Measurement of free radicals in vivo differs from practical analysis and is a problem. We no longer use the final product marker of the oxidation damage process Figure 1. Damage due to the oxidation process should use the nucleic acid quantity measurement and the causal damage using Cornet assay. Cornet assay is the end product of lipid peroxidase or protein oxidation. ${ }^{4}$ Free radicals are molecules or chains of molecules containing one or more unpaired chain whose orbital spacing are on the outer layer so they can be free to stick with other molecules. ROS is an oxygen derivative in the form of radical oxyen ehile RNS is a non-active radicals that can oxidize another atom or molecules and is very easy to be radical. Some kind of free radicals including: superoxide $\left(\mathrm{O}_{2}{ }^{-}\right)$, hydroxyl $\left(\mathrm{OH}^{-}\right)$, peroxyl $\left(\mathrm{RO}_{2}{ }^{\bullet}\right)$, hydroperoxyl $\left(\mathrm{HO}_{2}{ }^{\circ}\right)$, alkoxyl $(\mathrm{RO} \bullet)$, peroxyl $(\mathrm{ROO} \bullet)$, nitric oxide $(\mathrm{NO} \bullet)$, nitrogen dioxide $\left(\mathrm{NO}_{2}{ }^{\circ}\right)$ and lipid peroxyl $(\mathrm{LOO} \bullet)$ and non-radicals such as hydrogen peroxide $\left(\mathrm{H}_{2} \mathrm{O}_{2}\right)$, hypochlorous acid $(\mathrm{HOCl})$, ozone $\left(\mathrm{O}_{3}\right)$, singlet oxygen $(1 \Delta \mathrm{g})$, peroxynitrate $\left(\mathrm{ONOO}^{-}\right)$, nitrous acid $\left(\mathrm{HNO}_{2}\right)$, dinitrogen trioxide $\left(\mathrm{N}_{2} \mathrm{O}_{3}\right)$, lipid peroxide $(\mathrm{LOOH})$. In our body there are endogenous and exogenous source of free radicals. ${ }^{5,6}$ They can be produced by XO enzimes, NADPH oxidase and the others or by auto-oxidation such as adrenaline, dopamine and others, or by electron release from mitochondrial. The use of chemicals cush as doxorubicin, cigarettes etc, free transition metals catalytics (e.g Fe ${ }^{++}$, $\mathrm{Cu}^{+}$etc.) and environtmental radiation (such as radon, $\mathrm{UV}$, and others) may also causing the increase of body's oxydants. ${ }^{6-9}$ Reactive oxygen molecules such as superoxide $\left(\mathrm{O}_{2}, \mathrm{OOH}^{-}\right)$hydroxyl $\left(\mathrm{OH}^{-}\right)$and peroxyl $\left(\mathrm{ROOH}^{-}\right)$play a major and important role as oxidative stress in the pathogenesis of various disease. These free radicals are generated by:

A. mitochondria (superoxide radical and hydrogen peroxide) 
B. phagocytes (generators of nitric oxide and hydrogen peroxide during the 'respiratory burst' that takes place in activated phagocytic cells in order to kill bacteria after phagocytosis)

C. peroxisomes or microbodies (degrade fatty acids and other substances yielding hydrogen peroxide) and cytochrome P450 enzymes, responsible for many oxidation reactions of endogenous substrates

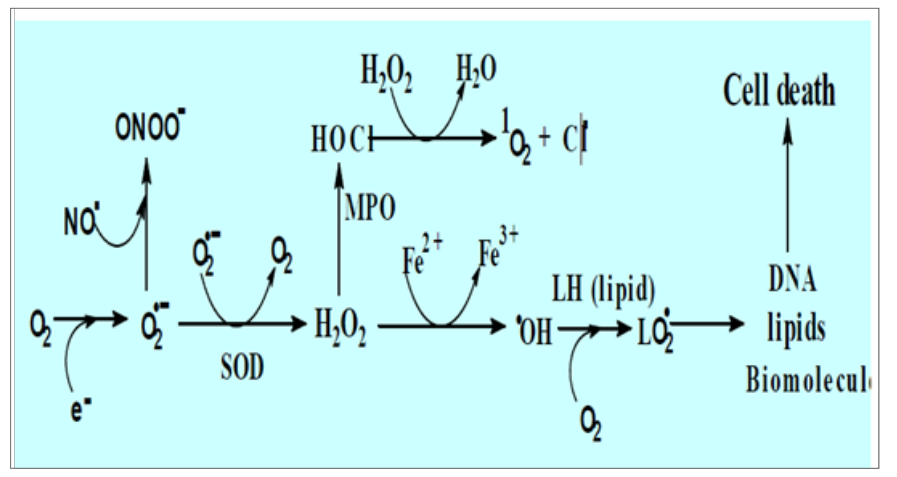

Figure I Figure of reactive oxygen species (ROS) and reactive nitrogen species (RNS). ${ }^{6}$

\section{Aging}

Aging process is a natural process. Genetic factor plays a role in the aging process through complex mechanism. The age differences among species is because of the genetic factor but this theory undergoing an evolution that suggest aging is not programmed and not genetic but caused by the accumulation of somatic destruction that can not be maintained or repaired. Genes control the activity level such as DNA repair or defence activity by removing antioxidants. This is the thing that control the life expectancy of a person. There is the possibility of slow gene disorders due to natural selection or gene adaptation in response to environmental changes such as changes in the quality of food intake Figure 2. Free radicals play a role throughout human life. At the embrio periods, free radical plays a role in stimulating the differentiation of cell and organs, on the growth period plays a role in the process of development and maturation of cells and organs only in the next stage free radicals play a role in aging process and the occurence of various degenerative diseases. The fundamental difference in the effects of free radicals on the growth period and adulthood are the accumulation of metabolic wastes, the remodelling process, cell damages and finally diseases as seen in Table 1. In the last two decades there have been many studies shows the antioxidant roles in health. The use of antioxidant or diet theraphy that contains many antioxidants can inhibit the development of several disease including aging process. Through a better research, it was found that many successfull antioxidants dan diet theraphy but many have failed to prove it. For example, research on the intake of nutrients that contain many vegetables and fruits can reduce cancer risk due to increased level sof beta caroten in the blood. But, theraphy that using beta carotene suplementation was not able to prove the effects. High consumption of vegetables and fruits can lower the level of free radicals in the body that will damage DNA but the suplemen theraphy of ascorbate, vitamin $\mathrm{E}$ and betacaroten was not able to reduce DNA damage that occured. Another well known study is $\mathrm{CHAOS}$ that can prove that vitamin $\mathrm{E}$ suplementation are protective against the cardiovascular events but this study can not be proven in the GISSI prevention trial study. ${ }^{10-14}$

Table I Differences in the effect of free radicals in young and adulthood ${ }^{4}$

\begin{tabular}{lll}
\hline \multirow{2}{*}{$\begin{array}{l}\text { Level of } \\
\text { effect }\end{array}$} & Timing of effect & \\
\cline { 2 - 3 } & During development & During adulthood \\
\hline & Mutation & Mutation \\
& Epimutation & Epimutation \\
Molecular & Modification (e.g. & Errors in biosynthesis \\
& glycosylation, cross-linkage) & $\begin{array}{l}\text { Modification (e.g. } \\
\text { linkage) }\end{array}$ \\
& Damage (e.g. oxidation) & Damage (e.g. oxidation) \\
& Diffusion (Brownian motion) & Diffusion (Brownian \\
& motion) \\
& Molecular interactions & Molecular interactions \\
& Differentiation (cell fate) & Differentiation (cell fate) \\
& Random segregation of & Random segregation of \\
constituents between & constituents between \\
daughter cells at division & daughter cells at division \\
& Cell-cell contacts & Cell-cell contacts \\
& Cell death & Cell death \\
& Cell migration & Cell migration
\end{tabular}

Accumulation of metabolic wastes

Cell number variation Tissue remodeling

Organ/ Size variation Cell loss

system Internal organization Injury due to toxins,

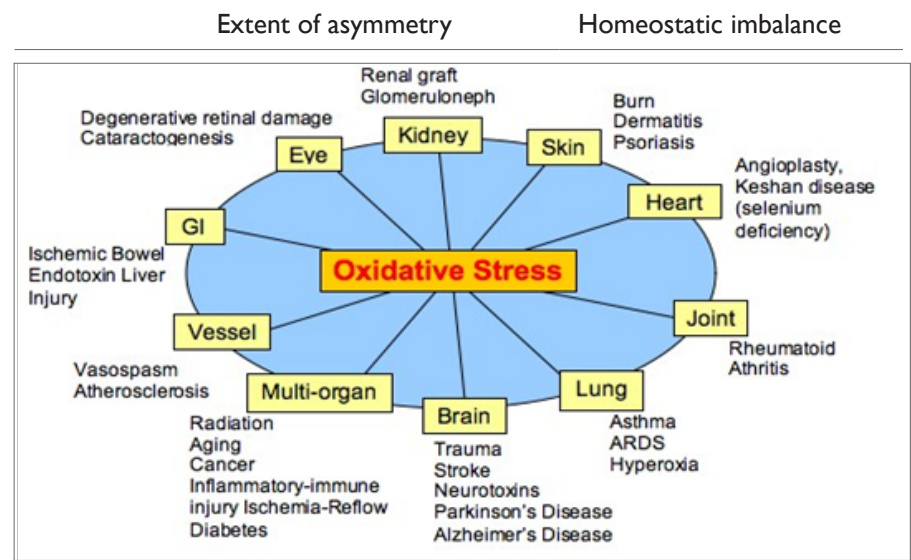

Figure 2 Stress oxidative effects on all organ. ${ }^{4}$

There are two things that can explain this controversy:

A. The protection effects possessed by the diet is not the same as the effect of suplementation theraphy.

B. Most of the free radicals require antioxidants that work on decreasing the oxidant effect. The power to decrease the oxidant effect in vitro is the total power of the antioxidants. 
C. The balance process of antioxidants and free radicals occur dinamically in the body. Low levels of antioxidant can stimulate the proliferation of cells. Increase in the oxidation can causes apoptosis whereas if the level is too high it can stop the apoptosis through the caspase enzyme.

\section{Physical Activity}

Increased production of free radicals during physicals exercise is caused by several factors, such as the increased of cathecholamine resulting in autooxidation, hypoxia and muscle reoxygenation, lactic acid that trigger the release of iron from myoglobin, and inflammation that occur due to impaired function of leukocytes. But there are still limited data that examine the acute and chronic effect of physical exercise in the aging process both in animals and humans. ${ }^{15}$ Physical activity and excercise is highly recommended to maintain the optimal health and prevent the degenerative disease Figure 3. Physical activities in the middle age, smoking cessation, maintain the blood pressure, and avoid obesity are the factors that independently associated with the decrease of cardiovascular event and lower overall mortality. Regular physical activity can restore the old age body composition in which the muscle mass increases and reduce fat mass and prevents some disease such as diabetes, hypertension, cancer and osteoporosis. Physical activity although producing free radicals but on the other hand can trigger the cell adaptation to increase the production of endogen antioxidant. Physical activities may increase endogenous antioxidant production in both young and old age. Although antioxidant activity can be improved with physical activity but the overall aging process leads to further decline in antioxidant activity. A sudden physical activity can increase the antioxidant activity in the skeletal muscle, heart and liver with different quality for both enzime antioxidant group or non-enzimatic antioxidant group. A gradual and continuous physical activity can increase the antioxidant enzime activity eventhough in the muscle that already aged. ${ }^{7,15}$

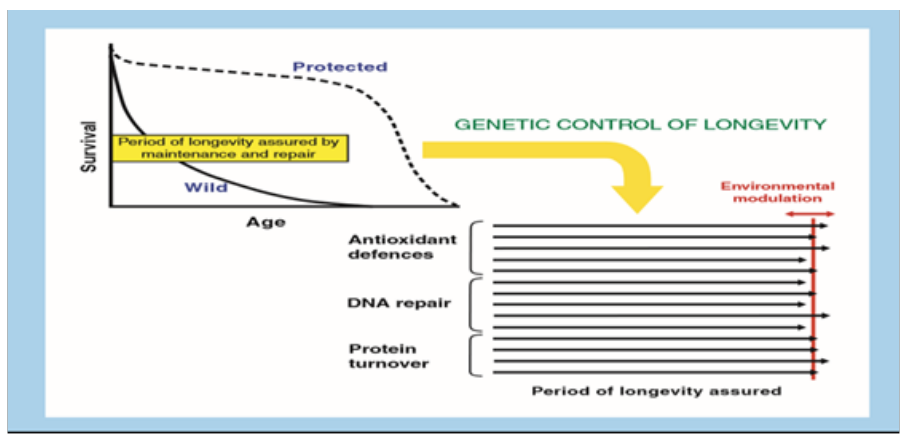

Figure 3 The influence of genetic and environmental factors on the aging process. $^{3}$

\section{Role of antioxidants}

Antioxidants are substrates that may inhibit or slow down the oxidation process. Endogenous antioxidants are in the form of non-enzymatic (such as uric acid, gluthathione, bilirubin, thiols, albumin, and nutrition factors: vitamin \& phenols) and enzymatic (such as superoxide dismutases, gluthathione peroxidases [GSHPx], \& catalase). In the normal subjects, endogenous antioxidants can maintain the balance with ROS but there are about $1 \%$ that can not be neutralizes by the endogenous antioxidants. Nutritional antioxidants is a source of antioxidants that important with a different mechanism for each type but mainly as free radical scavenger: directly neutralize, decrease peroxide concentration and improve the oxidized membrane, decrease ROS production, through lipid metabolism, short-chain free fat acid, and cholesteryl esters neutralize ROS. The body antioxidant ability can be estimated by measuring the levels of plasma antioxidants (micronutrients, enzyme, and the other antioxidants), but it should be remembered that it is just the content of the blood stream and may be not similar with the cells and tissues. Another thing that should be concern about is nutritional antioxidants administration can turn into a pro-oxidant. Several studies have been proved this to happen. Antioxidant supplementation should be cautiously administered, should be used when there is suspicion of excessive free radicals either due to environtmental exposure or diseases. Nutritional antioxidants often indicated to improve health status and prolong life expectancy. But there are still very few studies that prove the protective effect of micronutrients or nutritional antioxidants specifically. It is also unclear to be proven that the benefits a high vegetables and fruits diets for health can be replaced by antioxidant suplementation. Some important obstacles to prove the role of antioxidants in aging process are the lack of literatures about how the antioxidant mechanism of action and how to evaluate the marker of antioxidant theraphy. Antioxidant theraphy may need to be given earlier. Differences between the observational and clinical studies will lead to the differentiation of the exposure periods in antioxidants diet. Another important things are the animal studies, alhough studies are based in bilogical mechanism but when applied to human there will be changes or translations of results. Antioxidants have therapeutic doses individually, and a combined administration may change the therapeutic windows. Therapeutic doses can not be ascertained yet, excessive dose may even give the opposite effects. The presence of new antioxidants gives hopes that this suplementation can work better. Almost all previous studies used a nutritional antioxidant that classified as a non-enzymatic antioxidants. This antioxidants potentially become pro-oxidants while the enzimatic antioxidants are expected to work better and not become pro-oxidants in the body. Not many studies focusing on enzymatic antioxidants. One of the examples is sodium dismutase (SOD). SOD is also classified as primary and endogenous antioxidants. A better studies needed to test the effectiveness of this antioxidants. The things to be prepared are understanding the basic mechanism of aging process is a mandatory, how interrelated relationships between different antioxidants, the relationship between antioxidant and prooxidant factors, the pathogenesis of oxidation causing cell damage and diseases, and the discovery of antioxidant marker that believe to play a role in the real process. ${ }^{15-17}$

\section{Acknowledgements}

None.

\section{Conflict of interest}

The authors declared that no conflict of interest exists.

\section{References}

1. Fusco D, Colloca G, Monaco MLR, et al. Effects of antioxidant supplementation on the aging process. Clinical Interventions in Aging. 2007;2(3):377-387. 
2. Kirkwood TBL. Understanding Ageing From An Evolutionary Perspective. Journal of Internal Medicine. 2008:(263);117-127.

3. Couteur DGL, Lachlan AJ, Quinn JJ, et al. Aging biology and novel targets for drug discovery. J Gerontol A Biol Sci Med Sci. 2012;67(2):168-174.

4. Kregel $\mathrm{KC}$, Zhang HJ. An integrated view of oxidative stress in aging: basic mechanisms, functional effects, and pathological considerations. Am J Physiol Regul Integr Comp Physiol. 2007;(292):R18-R36.

5. Cutler RG. Antioxidants and aging. Am J Clin Nutr. 1991;(53):373S-379S

6. Mandal S, Yadav S, Yadav S, et al. Antioxidants: A Review. Journal of Chemical and Pharmaceutical Research. 2009;1(1):102-104.

7. Jones DP. Radical-free biology of oxidative stress. Am J Physiol Cell Physiol. 2008;(295):C849-C868.

8. Shinde A, Ganu J , Naik P. Effect of Free Radicals \& Antioxidants on Oxidative Stress: A Review. Journal of Dental \& Allied Sciences. 2012;1(2):63-66.

9. Pham-Huy LA, He H, Pham-Huy C. Free Radicals, Antioxidants in Disease and Health. Int J Biomed Sci. 2008;4(2):89-96.

10. Scandalios JG. Oxidative Stress: Molecular Perception And Transduction Of Signals Triggering Antioxidant Gene Defenses. Brazilian Journal of Medical and Biological Research. 2005;(38):995-1014.
11. Stephens NG, Parsons A, Schofield PM, et al. Randomised controlled trial of vitamin $\mathrm{E}$ in patients with coronary disease: Cambridge Heart Antioxidant Study (CHAOS). Lancet. 1996;(347):781-786.

12. Dietary supplementation with $n-3$ polyunsaturated fatty acids and vitamin E after myocardial infarction: results of the GISSI-Prevenzione trial. Lancet 1999;(354):447-55.

13. Fraga CG, Motchnik PA, Wyrobek AJ, et al. Smoking and low antioxidant levels increase oxidative damage to sperm DNA. Mutat Res. 1996;(351):199-203.

14. Benzie IF, Strain JJ. Ferric reducing/antioxidant power assay: direct measure of total antioxidant activity of biological fluids and modified version for simultaneous measurement of total antioxidant power and ascorbic acid concentration. Meth Enzymol. 1999;(299):15-27.

15. Joe Beckman. The ABC's of the Reactions between Nitric Oxide, Superoxide, Peroxynitrite and Superoxide Dismutase. Oxygen'99: USA; $1: 13$

16. Barry Halliwell. The antioxidant paradox. Lancet. 2000;(355):1179-1180.

17. Sujogya KP. Assay Guided Comparison for Enzymatic and NonEnzymatic Antioxidant Activities with Special Reference to Medicinal Plants. OAPR. 2012. 